\section{Cytotoxic Norhopene Triterpenoids from the Bark of Exothea paniculata from Abaco Island, Bahamas}

\section{Bhuwan K. Chhetri, Noura S. Dosoky, William N. Setzer}

Department of Chemistry, University of Alabama in Huntsville, Huntsville, AL, USA

\section{Abstract \\ $\nabla$}

Bioactivity-directed preparative chromatography of the cytotoxic crude dichloromethane bark extract of Exothea paniculata has yielded two new cytotoxic norhopene triterpenoids, exotheol A and exotheol $\mathrm{B}$. The structures were determined by nuclear magnetic resonance and high-resolution mass spectrometry and were found to be potently cytotoxic to MCF-7 and 5637 cells in vitro.

\section{Key words}

Exothea paniculata - Sapindaceae $\cdot$ norhopene $\cdot$ triterpenoid cytotoxicity

Exothea paniculata (Juss.) Radlk. (Sapindaceae), "butter bough”, is a medium-sized tree with bipinnate leaves arranged alternately with 4-6 lanceolate leaflets; infloresences are corymbose-paniculate and the fruit is a dark purple drupe [1]. The tree ranges from Florida, the Caribbean, and Mexico, south through Central America to Colombia and Ecuador [2]. As far as we are aware, the only documented ethnobotanical use of this plant is for tanning animal hides in Caoba, Guatemala [3]. There are apparently no reported medicinal uses of this tree nor have there been any phytochemical investigations. In the course of our study on the biological activities of plants from Abaco Island, Bahamas [4-6], we have examined the crude bark extract of E. paniculata and found it to show considerable cytotoxicity.

We had previously found the crude acetone bark extract of $E$. paniculata from Abaco Island to show selective in vitro cytotoxic activity to 5637 human bladder carcinoma cells, but little or no activity toward SK-Mel-28 (human melanoma), Hep-G2 (human hepatocellular carcinoma), or MDA-MB-231 (human mammary adenocarcinoma) cells [6]. The dried bark from E. paniculata was extracted with dichloromethane to give a crude extract with a $1.68 \%$ yield. Bioactivity-directed preparative chromatographic separation of the crude bark extract led to the isolation and characterization of two new cytotoxic norhopene triterpenoids, exotheol A and exotheol B ( $\odot$ Fig. 1). Exotheol A was isolated as a light yellow powder, which had a molecular formula $\mathrm{C}_{36} \mathrm{H}_{50} \mathrm{O}_{5}$ based on electron spray ionization high-resolution mass spectrometry (obsd. $[\mathrm{M}-\mathrm{H}]^{-}$at $m / z$ 561.3549, calcd. $[\mathrm{M}-\mathrm{H}]^{-} 561.3580$ ), ${ }^{1} \mathrm{H}$, ${ }^{13} \mathrm{C}$, and gHSQC NMR spectral data ( Table 1 ). The proton spectrum showed a characteristic feature of a triterpenoid with five methyl signals ( $\delta_{\mathrm{H}}: 0.65,0.93,0.96,1.43$, and $1.63 \mathrm{ppm}$ ). A downfield methyl shift of $1.63 \mathrm{ppm}$ along with a broad singlet at $4.66 \mathrm{ppm}\left(\mathrm{CH}_{2}\right)$ was indicative of an isopropenyl group. With a terminal vinyl group at $\delta_{\mathrm{H}}: 5.10,5.43 \mathrm{ppm}$, these features suggested a hopene skeleton based on a structure similarity search using the Dictionary of Natural Products [7]. The compound had many similarities in terms of its NMR data to hopene derivatives

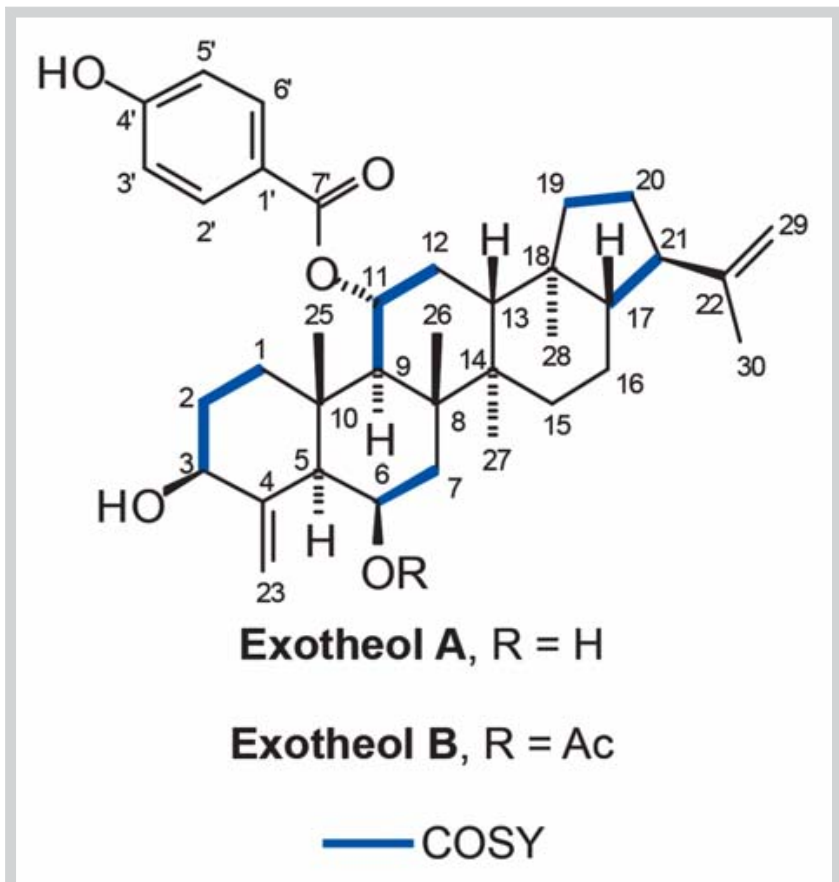

Fig. 1 Structures of exotheols A and B, including numbering scheme and important COSY correlations.

known as the cavalerols [8]. Five primary, ten secondary, twelve tertiary, and nine quaternary carbon atoms accounted for a total of thirty-six carbon atoms in the molecular formula. ${ }^{1} \mathrm{H},{ }^{1} \mathrm{H}-\mathrm{COSY}$ correlations established the spin system sequence from $\mathrm{H}-1$ to $\mathrm{H} 2$ and $\mathrm{H}-3, \mathrm{H}-6$ to $\mathrm{H}-7, \mathrm{H} 9$ to $\mathrm{H}-11$ and $\mathrm{H}-12, \mathrm{H}-19$ to $\mathrm{H}-20$, and $\mathrm{H} 17$ to $\mathrm{H} 21$. These fragments were put into place based on HMBC correlations from the methyl groups as well as the $\mathrm{CH}$ groups present at the ring junctions. Carbon chemical shift and IR data indicated the presence of two hydroxyl groups. Their position and stereochemistry were ascertained as C-3 and C- 6 based on COSY, HMBC, and ROESY data ( Table 2). Both OH groups at C-3 and $\mathrm{C}-6$ had $\beta$ configuration established through the ROESY correlations between $\mathrm{H}_{\alpha}-3, \mathrm{H}_{\alpha^{-}}-5$ and $\mathrm{H}_{\alpha}-6, \mathrm{H}_{\alpha^{-}}-5$. Proton signals at $\delta$ $6.85(\mathrm{~d}, J=8.75 \mathrm{~Hz})$ and $\delta 7.79(\mathrm{~d}, J=8.75 \mathrm{~Hz})$ along with carbon chemical shift values $\delta=120.93,131.52,115.36,161.95$, and 164.51 and IR, suggested the presence of a p-hydroxybenzoate group, a functionality present in similar norhopene compounds in a previous study as well [9]. The critical position of the p-hydroxybenzoate group was obvious due to its $\mathrm{H}-11$ lying in the $\mathrm{H}-$ 9 to $\mathrm{H}-11$ and $\mathrm{H}-12$ COSY spin system, the $\mathrm{H}-2^{\prime}, 6^{\prime}$ of the benzene ring showing a ROESY correlation to $\mathrm{H}_{\beta^{-}}-1\left(\delta_{\mathrm{H}}: 1.98-2.03, \mathrm{~m}\right)$, and HMBC correlations between $\mathrm{C}-7$ ' and $\mathrm{H}-11$. The $\alpha$ configuration of the p-hydroxybenzoate group was further supported by the ROESY correlation between $\mathrm{H}_{\beta}-11$ and Me-25.

Similarly, exotheol B ( $\bullet$ Fig. 1) was an analog of exotheol A with the only difference in the presence of an acetoxy group at C-6 instead of a hydroxyl group as found in exotheol A. The acetoxy group had a $\beta$ configuration, supported by the ROESY correlation between $\mathrm{H}-6$ and $\mathrm{H}-5$. Here, too, the $\mathrm{OH}$ group at $\mathrm{C}-3$ had a $\beta$ configuration whereas the p-hyroxybenzoate group was at $\mathrm{C}-11$ with $\mathrm{H}-2$ ', 6' showing ROESY correlations to $\mathrm{H}_{\beta}-1\left(\delta_{\mathrm{H}}\right.$ : 2.07-2.12, $\mathrm{m})$. The major COSY correlations, HMBC, ROESY, and other spectral data supporting the structure are summarized in $\odot$ Table 2 and Fig. 1. 


\begin{tabular}{|c|c|c|c|c|c|}
\hline Position & $\delta^{13} \mathrm{C}$ & & $\delta^{1} \mathrm{H}$ & Кеу НМBC & Key ROESY \\
\hline 1 & 41.95 & $\mathrm{CH}_{2}$ & $\begin{array}{l}1.98-2.03(\mathrm{~m}), 1.29 \\
\text { (overlaid) }\end{array}$ & $C-2, C-3, C-5, C-10$ & $\mathrm{H}-2^{\prime}, 6^{\prime}, \mathrm{H}-25$ \\
\hline 2 & 32.86 & $\mathrm{CH}_{2}$ & $1.55,1.13$ (overlaid) & & \\
\hline 3 & 71.49 & $\mathrm{CH}$ & $3.67-3.72(\mathrm{~m})$ & & $\mathrm{H}-5$ \\
\hline 4 & 151.66 & quat & & & \\
\hline 5 & 51.12 & $\mathrm{CH}$ & $1.61(\mathrm{~s})$ & $\begin{array}{l}\text { C-25, C-10, C-3, C-23, } \\
\text { C-4, C-6 }\end{array}$ & $\mathrm{H}-9, \mathrm{H}-6$ \\
\hline 6 & 67.90 & $\mathrm{CH}$ & $4.15(\mathrm{~m})$ & $C-8, C-10$ & $\mathrm{H}-5, \mathrm{H}-7$ (1.45), H-23 \\
\hline 7 & 39.50 & $\mathrm{CH}_{2}$ & $1.62,1.45$ (overlaid) & & \\
\hline 8 & 42.96 & quat & & & \\
\hline 9 & 50.62 & $\mathrm{CH}$ & $1.89(\mathrm{~d}, J=11.5 \mathrm{~Hz})$ & $\begin{array}{l}\text { C-25, C-26, C-12, C-10, } \\
\text { C-1, C-8, C-11 }\end{array}$ & $\begin{array}{l}\mathrm{H}-25, \mathrm{H}-26, \mathrm{H}-13, \\
\mathrm{H}-27, \mathrm{H}-1\end{array}$ \\
\hline 10 & 39.29 & quat & & & \\
\hline 11 & 72.56 & $\mathrm{CH}$ & $5.51(\mathrm{~m})$ & $C-7^{\prime}, C-9$ & $\mathrm{H}-25, \mathrm{H}-26, \mathrm{H}-13$ \\
\hline 12 & 31.19 & $\mathrm{CH}_{2}$ & $1.69,1.59$ (overlaid) & & \\
\hline 13 & 45.69 & $\mathrm{CH}$ & $\begin{array}{l}1.68 \text { (overlaid) (1.67- } \\
1.71 \text { ) }\end{array}$ & $\begin{array}{l}\text { C-28, C-27, C-12, C-14, } \\
\text { C-18 }\end{array}$ & $\mathrm{H}-11$ \\
\hline 14 & 41.67 & quat & & & \\
\hline 15 & 31.98 & $\mathrm{CH}_{2}$ & $\begin{array}{l}1.31,1.18 \text { (overlaid) } \\
(1.29-1.32,1.17-1.20)\end{array}$ & $C-27, C-14$ & \\
\hline 16 & 20.62 & $\mathrm{CH}_{2}$ & $1.35,1.16$ (overlaid) & & \\
\hline 17 & 53.51 & $\mathrm{CH}$ & 1.02 (overlaid) & $\begin{array}{l}\text { C-20, C-19, C-18, C-13, } \\
\text { C-21 }\end{array}$ & \\
\hline 18 & 43.51 & quat & & & \\
\hline 19 & 39.29 & $\mathrm{CH}_{2}$ & $1.41,1.05$ (overlaid) & & \\
\hline 20 & 26.76 & $\mathrm{CH}_{2}$ & $\begin{array}{l}1.75-1.83(\mathrm{~m}), 1.38 \\
\text { (overlaid) }\end{array}$ & C-17, C-21, C-19 & $\mathrm{H}-21$ \\
\hline 21 & 47.23 & $\mathrm{CH}$ & $2.21(\mathrm{dt}, J=5 \mathrm{~Hz}, 10 \mathrm{~Hz})$ & $C-30, C-20, C-17$ & $\mathrm{H}-28, \mathrm{H}-20(1.75-1.83)$ \\
\hline 22 & 147.21 & quat & & & \\
\hline 23 & 105.13 & $\mathrm{CH}_{2}$ & $5.1,5.43$ (bs) & $C-5, C-3, C-4$ & $\mathrm{H}-6$ \\
\hline 25 & 16.05 & $\mathrm{CH}_{3}$ & $0.93(s)$ & $C-10, C-1, C-5$ & $\mathrm{H}-26, \mathrm{H}-11$ \\
\hline 26 & 18.00 & $\mathrm{CH}_{3}$ & $1.43(\mathrm{~s})$ & C-10, C-14, C-8, C-9 & $\mathrm{H}-11, \mathrm{H}-25$ \\
\hline 27 & 16.59 & $\mathrm{CH}_{3}$ & $0.96(s)$ & C-15, C-13, C-14 & $\mathrm{H}-28, \mathrm{H}-9$ \\
\hline 28 & 14.60 & $\mathrm{CH}_{3}$ & $0.65(s)$ & C-17, C-13, C-18, C-19 & $\mathrm{H}-21$ \\
\hline 29 & 110.07 & $\mathrm{CH}_{2}$ & 4.66 (bs) & $C-30, C-21$ & $\mathrm{H}-30$ \\
\hline 30 & 19.26 & $\mathrm{CH}_{3}$ & $1.63(\mathrm{~s})$ & $C-21, C-29, C-22$ & \\
\hline $1^{\prime}$ & 120.93 & quat & & & \\
\hline $2^{\prime}, 6^{\prime}$ & 131.52 & $\mathrm{CH}$ & $7.79(\mathrm{~d}, J=8.75 \mathrm{~Hz})$ & $C-3^{\prime}, 5^{\prime}, C-4^{\prime}, C-7^{\prime}$ & $\mathrm{H}-1$ \\
\hline $3^{\prime}, 5^{\prime}$ & 115.36 & $\mathrm{CH}$ & $6.85(\mathrm{~d}, J=8.75 \mathrm{~Hz})$ & $C-1^{\prime}, C-4^{\prime}$ & \\
\hline $4^{\prime}$ & 161.95 & quat & & & \\
\hline $7^{\prime}$ & 164.51 & quat & & & \\
\hline
\end{tabular}

The two compounds were screened for in vitro cytotoxic activity against MCF-7 (human breast adenocarcinoma) and 5637 (human bladder carcinoma) cells ( Table 3 ). It is difficult to speculate about the biomolecular target(s) of the exotheols, but several cytotoxicity-relevant molecular targets have been targeted by pentacyclic triterpenoids [10], including topoisomerase II [11, 12], farnesyl protein transferase [13], DNA polymerase $\beta$ [14], and lipoxygenase [15]. In order to provide some insight, a molecular docking analysis of exotheol A, exotheol B, and ursolic acid (a triterpenoid known to inhibit topoisomerase II, DNA polymerase $\beta$, and lipoxygenase) was carried out using the Molegro Virtual Docking program $[16,17]$. The docking energies of the lowest-energy docked poses are summarized in Table 4. Except for the ATP binding site of topoisomerase II, exotheol A and exotheol B both docked more strongly to the protein targets examined than did ursolic acid. This docking study suggests that the DNA binding site of topoisomerase II could be the protein target for the exotheols.

There are only a few hopene triterpenoids described in the literature (around 38 are listed in the Dictionary of Natural Products [7]) and most of these do not include biological activities. In this present work, we have presented the considerable cytotoxic activities of two norhopene triterpenoids from the bark of E. paniculata.

\section{Materials and Methods}

General experimental procedures

NMR spectra were obtained by a Varian INOVA 500 spectrometer for ${ }^{1} \mathrm{H}$ NMR, ${ }^{13} \mathrm{C}$ NMR, COSY, ROESY, HSQC, and HMBC. HRMS/ESI were measured with a Bruker microOTO-Q II spectromer. IR spectra were obtained with a Perkin-Elmer Spectrum One FT-IR spectrophotometer.

\section{Plant material}

The stem bark of E. paniculata was collected on June 18, 2002, from a mature tree growing in Marsh Harbour, Abaco Island, Bahamas $\left(26^{\circ} 32.21{ }^{\prime} \mathrm{N}, 77^{\circ} 3.21^{\prime} \mathrm{W}, 7.9 \mathrm{~m}\right.$ asl $)$. The plant was identified by W.N. Setzer by comparison with herbarium samples from Fairchild Tropical Garden Herbarium (FTG) and the Herbarium of the National Trust for the Cayman Islands (CAYM). A voucher specimen has been deposited in the University of Alabama in 


\begin{tabular}{|c|c|c|c|c|c|}
\hline $\begin{array}{l}\text { Posi- } \\
\text { tion }\end{array}$ & $\delta^{13} \mathrm{C}$ & & $\delta^{1} \mathrm{H}$ & Кеу НMBC & Key ROESY \\
\hline 1 & 41.63 & $\mathrm{CH}_{2}$ & 2.07-2.12 (m), 1.36 (overlaid) & C-2, C-10, C-9 & $\mathrm{H}-25$ \\
\hline 2 & 32.41 & $\mathrm{CH}_{2}$ & $1.59,1.36$ (overlaid) & & \\
\hline 3 & 71.17 & $\mathrm{CH}$ & $3.71-3.78(\mathrm{~m})$ & $C-2, C-23$ & $\begin{array}{l}\mathrm{HO}-3, \mathrm{H}-5, \mathrm{H}-2 \\
(1.59)\end{array}$ \\
\hline 4 & 150.84 & quat & & & \\
\hline 5 & 49.25 & $\mathrm{CH}$ & $1.96(\mathrm{~s})$ & & $\mathrm{H}-6, \mathrm{H}-3, \mathrm{H}-27$ \\
\hline 6 & 71.51 & $\mathrm{CH}$ & $5.10-5.13(\mathrm{~m})$ & C- $10, C-8$ & $H-7(1.52-1.56, d, d)$ \\
\hline 7 & 35.38 & $\mathrm{CH}_{2}$ & $\begin{array}{l}1.52-1.56(\mathrm{~d}, \mathrm{~d}), 1.78 \text { (overlaid) } \\
(11=15 \mathrm{~Hz}, / 2=2.15 \mathrm{~Hz})\end{array}$ & C-26, C-8, C-5, C-9, C-C-6 & \\
\hline 8 & 42.63 & quat & & & \\
\hline 9 & 50.10 & $\mathrm{CH}$ & 1.97 (overlaid) & & \\
\hline 10 & 39.29 & quat & & & \\
\hline 11 & 72.26 & $\mathrm{CH}$ & $5.46-5.58(\mathrm{~m})$ & C-10, C-9, C-7' & $\mathrm{H}-25, \mathrm{H}-26, \mathrm{H}-13$ \\
\hline 12 & 31.22 & $\mathrm{CH}_{2}$ & 1.62-1.71 (overlaid) & & \\
\hline 13 & 45.87 & $\mathrm{CH}$ & 1.69-1.72 (overlaid) & C-12, C-14, C-18, C-9, C-11 & \\
\hline 14 & 41.81 & quat & & & \\
\hline 15 & 31.89 & $\mathrm{CH}_{2}$ & $\begin{array}{l}1.13-1.19 \text { (overlaid), } 1.25 \\
\text { (overlaid) }\end{array}$ & & \\
\hline 16 & 20.54 & $\mathrm{CH}_{2}$ & $\begin{array}{l}1.32-1.36 \text { (overlaid), } 1.16 \\
\text { (overlaid) }\end{array}$ & & \\
\hline 17 & 53.38 & $\mathrm{CH}$ & $1.01-1.06(\mathrm{~m})$ & & \\
\hline 18 & 43.47 & quat & & & \\
\hline 19 & 39.29 & $\mathrm{CH}_{2}$ & $1.05-1.08(\mathrm{~m}), 1.4$ (overlaid) & & \\
\hline 20 & 26.73 & $\mathrm{CH}_{2}$ & $1.78,1.38$ (overlaid) & & \\
\hline 21 & 47.19 & $\mathrm{CH}$ & $2.17-2.24(\mathrm{~m})$ & C-30, C-20, C-29, C-22, C-17 & $\mathrm{H}-28, \mathrm{H}-29$ \\
\hline 22 & 147.28 & quat & & & \\
\hline 23 & 104.02 & $\mathrm{CH}_{2}$ & $4.54,5.09(\mathrm{~s})$ & $C-5, C-3, C-4$ & \\
\hline \multicolumn{6}{|l|}{24} \\
\hline 25 & 15.90 & $\mathrm{CH}_{3}$ & $0.96(s)$ & $C-10, C-1, C-5, C-9$ & $\mathrm{H}-11$ \\
\hline 26 & 18.04 & $\mathrm{CH}_{3}$ & $1.26(\mathrm{~s})$ & C-7, C-14, C-9, C-8 & \\
\hline 27 & 16.56 & $\mathrm{CH}_{3}$ & $0.99(s)$ & $\mathrm{C}-15, \mathrm{C}-14, \mathrm{C}-8, \mathrm{C}-13$ & $\mathrm{H}-28, \mathrm{H}-9$ \\
\hline 28 & 14.62 & $\mathrm{CH}_{3}$ & $0.65(s)$ & C-19, C-18, C-13, C-17 & $\begin{array}{l}\mathrm{H}-21, \mathrm{H}-19(1.4 \\
\text { overlaid) }\end{array}$ \\
\hline 29 & 110.10 & $\mathrm{CH}_{2}$ & $4.67(s)$ & $C-30, C-21$ & $\mathrm{H}-30, \mathrm{H}-21$ \\
\hline 30 & 19.24 & $\mathrm{CH}_{3}$ & $1.62(\mathrm{~s})$ & $C-21, C-29, C-22$ & $\mathrm{H}-29$ \\
\hline $1^{\prime}$ & 120.85 & quat & & & \\
\hline $2^{\prime}, 6^{\prime}$ & 131.77 & $\mathrm{CH}$ & $7.81(\mathrm{~d}, J=10 \mathrm{~Hz})$ & $C-3^{\prime}, 5^{\prime}, 4^{\prime}, 7^{\prime}$ & $\begin{array}{l}\mathrm{H}-1(2.07-2.12, \mathrm{~m}) \\
\mathrm{H}-3^{\prime}, 5^{\prime}\end{array}$ \\
\hline $3^{\prime}, 5^{\prime}$ & 115.43 & $\mathrm{CH}$ & $6.86(\mathrm{~d}, J=10 \mathrm{~Hz})$ & $C-2^{\prime}, 6^{\prime}, 1^{\prime}, 4^{\prime}$ & $\mathrm{H}-2^{\prime}, 6^{\prime}$ \\
\hline $4^{\prime}$ & 161.99 & $\mathrm{OH}$ & & & \\
\hline $7^{\prime}$ & 164.49 & quat & & & \\
\hline $\mathrm{AcO}$ & 21.42 & $\mathrm{CH}_{3}$ & $2.00(s)$ & Quat of AcO, C-6 & \\
\hline $\mathrm{AcO}$ & 169.87 & quat & & & \\
\hline
\end{tabular}

Table 2 NMR data for exotheol B. 


\begin{tabular}{|c|c|c|c|c|c|c|}
\hline \multirow[t]{3}{*}{ Ligand } & \multicolumn{2}{|c|}{ Topoisomerase II } & \multicolumn{2}{|c|}{ DNA Polymerase $\beta$} & \multirow{3}{*}{$\begin{array}{l}\text { 5-Lipoxy- } \\
\text { genase } \\
3 \text { V99 }\end{array}$} & \multirow{3}{*}{$\begin{array}{l}\text { Farnesyl protein } \\
\text { transferase } \\
1 \mathrm{JCQ}\end{array}$} \\
\hline & 1QZR & 2RGR & 2BPC & $3 U X N$ & & \\
\hline & ATP site & DNA site & & & & \\
\hline Exotheol A & -53.5 & -205.4 & -93.3 & -84.9 & -104.0 & -92.6 \\
\hline Exotheol B & -62.2 & -204.3 & -99.3 & -91.5 & -109.2 & -93.8 \\
\hline Ursolic acid & -57.7 & -149.6 & -73.4 & -70.9 & -79.8 & -78.5 \\
\hline
\end{tabular}

Table 4 MolDock molecular docking energies $(\mathrm{kJ} / \mathrm{mol})$ for norhopene triterpenoids from $E$. paniculata with antitumor relevant protein targets.
Superfraction 2 (combined Frs. 46-52, $217.40 \mathrm{mg}$ ) was further separated on a silica gel column $(50 \mathrm{~g}, 5-15 \mu \mathrm{m})$ eluting with $1: 1: 1$ mixture of hexane, $\mathrm{CH}_{2} \mathrm{Cl}_{2}$, and ethyl acetate followed by further purification using preparative TLC $(20 \times 20 \mathrm{~cm}$, plasticbacked, $200 \mu \mathrm{m}$ thickness) with $1.0: 1.0: 1.2$ (hexane: $\mathrm{CH}_{2} \mathrm{Cl}_{2}$ : EtOAc) to give exotheol $\mathrm{B}(41.08 \mathrm{mg})$ as a colorless powder. IR: 3445, 2941, 1694, 1610, 1265, 1239, $1166 \mathrm{~cm}^{-1}$; MP: $262^{\circ} \mathrm{C}$; HRMS/ESI, $m / z$ : obsd. [M - H] $]^{-}$603.3719, calcd. [M - H] 603.3685.

\section{Cytotoxicity screening}

The crude extract and purified compounds were tested for cytotoxicity against MCF-7 (human breast adenocarcinoma cells, ATCC No. HTB-22) and 5637 (human bladder carcinoma cells, ATCC No. HTB-9) cells. MCF-7 cells were grown in RPMI 1640 supplemented with 10\% fetal bovine serum (FBS), 30 mM HEPES, sodium bicarbonate, and penicillin-streptomycin. Then, 5637 cells were grown in RPMI 1640 supplemented with 10\% FBS, $1.0 \mathrm{mM}$ sodium pyruvate, $2.5 \mathrm{~g}$ glucose, $30 \mathrm{mM}$ HEPES, sodium bicarbonate, and penicillin-streptomycin. In vitro cytotoxic activity was performed using the 96-well MTT assay as previously reported [18].

\section{Computational methods}

Protein-ligand docking studies were carried out based on the crystal structures of yeast topoisomerase II (PDB 1QZR [19] and 2RGR [20]), rat DNA polymerase $\beta$ (PDB 2BPC [21] and 3 UXN [22]), human 5-lipoxygenase (PDB 3V99 [23]), and human farnesyl protein transferase (PDB 1JCQ [24]). Prior to docking, all solvent molecules and the cocrystallized ligands were removed from the structures. Molecular docking calculations for all of the compounds with each of the proteins were undertaken using Molegro Virtual Docker v. 6.0.1 [16,17] with a sphere large enough to accommodate the cavity centered on the binding sites of each protein structure in order to allow each ligand to search. If a co-crystallized inhibitor or substrate was present in the structure, then that site was chosen as the binding site. If no co-crystallized ligand was present, then suitably sized cavities were used as potential binding sites. Standard protonation states of the proteins based on a neutral pH were used in the docking studies. The protein was used as a rigid model structure; no relaxation of the protein was performed. Assignments of charges on each protein were based on standard templates as part of the Molegro Virtual Docker program; no other charges were necessary to be set. Each ligand structure was built using Spartan '14 for Windows [25]. The structures were geometry optimized using the MMFF force field [26]. Flexible ligand models were used in the docking and subsequent optimization scheme. Different orientations of the ligands were searched and ranked based on their energy scores. The RMSD threshold for multiple cluster poses was set at $<1.00 \AA$. The docking algorithm was set at maximum iterations of 1500 with a simplex evolution population size of 50 and a minimum of 100 runs for each ligand. Each binding site of oligomeric structures was searched with each ligand. The lowest-energy (strongest-docking) poses for each ligand in each protein target are summarized in 0 Table 4.

\section{Acknowledgments \\ $\nabla$}

We are grateful to Prof. Bernhard Vogler for technical assistance with NMR data collection and Ek Raj Thapaliya (Laboratory of Molecular Photonics, Department of Chemistry, University of Miami, Florida) for collection of HRMS data. We are very grateful to an anonymous private donor for generously funding this research.

\section{Conflict of Interest}

$\nabla$

The authors declare that they have no conflicts of interest.

\section{References}

1 Nickrent DL, Eshbaugh WH, Wilson TK. The vascular flora of Andros Island, Bahamas. Oxford, Ohio: Willard Sherman Turrell Herbarium, Miami University; 1991: 145-147

2 Tropicos.org. Missouri botanical garden. Available at http://www. tropicos.org/Name/28600911. Accessed September 6, 2015

3 Mutchnick PA, McCarthy BC. An ethnobotanical analysis of the tree species common to the subtropical most forests of the Petén, Guatemala. Econ Bot 1997; 51: 158-183

4 Schmidt JM, Noletto JA, Vogler B, Setzer WN. Abaco bush medicine: chemical composition of the essential oils of four aromatic medicinal plants from Abaco Island, Bahamas. J Herbs Spices Med Plants 2007; 12: 43-65

5 Monzote L, Piñón A, Setzer WN. Antileishmanial potential of tropical rainforest plant extracts. Medicines 2014; 1: 32-55

6 Setzer MC, Newby JS, Moriarity DM, Setzer WN. A phytopharmaceutical survey of Abaco, Island, Bahamas. Am J Essent Oils Nat Prod 2015; 2: 10-17

7 Dictionary of Natural Products on DVD, v. 23: 2. Boca Raton, Florida: CRC Press; 2015

8 Cheng L, Shen LM, Zhang M, Li N, Li X, Ma ZJ, Qu HB. Eleven new triterpenes from Eurycorymbus cavaleriei. Helv Chim Acta 2010; 93: 22632275

9 Chávez JP, David JM, Yang SW, Cordell GA. 24-Norhopene derivatives from Diatenopteryx sorbifolia. J Nat Prod 1997; 60: 909-911

10 Setzer WN, Setzer MC. Plant-derived triterpenoids as potential antineoplastic agents. Mini Rev Med Chem 2003; 3: 540-556

11 Moriarity DM, Huang J, Yancey CA, Zhang P, Setzer WN, Lawton RO, Bates $R B$, Caldera $S$. Lupeol is the cytotoxic principle in the leaf extract of Dendropanax cf. querceti. Planta Med 1998; 64: 370-372

12 Wada SI, Iida A, Tanaka R. Triterpene constituents from the stem bark of Pinus luchuensis and their DNA topoisomerase II inhibitory effect. Planta Med 2001; 67: 659-664

13 Sturm S, Gil RR, Chai HB, Ngassapa OD, Santisuk T, Reutrakul V, Howe A, Moss M, Besterman JM, Yang SL, Farthing JE, Tait RM, Lewis JA, O'Neill MJ, Farnsworth NR, Cordell GA, Pezzuto JM, Kinghorn AD. Lupane derivatives from Lophopetalum wallichii with farnesyl protein transferase inhibitory activity. J Nat Prod 1996; 59: 658-663

14 Chaturvedula VSP, Zhou BN, Gao Z, Thomas SJ, Hecht SM, Kingston DGI. New lupane triterpenoids from Solidago canadensis that inhibit the lyase activity of DNA polymerase $\beta$. Bioorg Med Chem 2004; 12 : 6271-6275 
15 Simon A, Najid A, Chulia AJ, Delage C, Rigaud M. Inhibition of lipoxygenase activity and HL60 leukemic cell proliferation by ursolic acid isolated from heather flowers (Calluna vulgaris). Biochim Biophys Acta 1992; 1125: 68-72

16 Molegro Virtual Docker v 6.0.0. Aarhus, Denmark: Molegro ApS; 2013

17 Thomsen R, Christensen MH. MolDock: a new technique for high-accuracy molecular docking. J Med Chem 2006; 49: 3315-3321

18 Setzer MC, Setzer WN, Jackes BR, Gentry GA, Moriarity DM. The medicinal value of tropical rainforest plants from Paluma, North Queensland, Australia. Pharmaceut Biol 2001; 39: 67-78

19 Classen S, Olland S, Berger JM. Structure of the topoisomerase II ATPase region and its mechanism of inhibition by the chemotherapeutic agent ICRF-187. Proc Natl Acad Sci U S A 2003; 100: 10629-10634

20 Dong KC, Berger JM. Structural basis for gate-DNA recognition and bending by type IIA topoisomerases. Nature 2007; 450: 1201-1205

21 Sawaya MR, Pelletier H, Kumar A, Wilson SH, Kraut J. Crystal structure of rat DNA polymerase beta: evidence for a common polymerase mechanism. Science 1994; 264: 1930-1935

22 Gridley CL, Rangarajan S, Firbank S, Dalal S, Sweasy JB, Jaeger J. Structural changes in the hydrophobic hinge region adversely affect the activity and fidelity of the I260Q mutator DNA polymerase $\beta$. Biochemistry 2013; 52: 4422-4432

23 Gilbert NC, Rui Z, Neau DB, Waight MT, Bartlett SG, Boeglin WE, Brash AR, Newcomer ME. Conversion of human 5-lipoxygenase to a 15-lipoxygenase by a point mutation to mimic phosphorylation at Serine-663. FASEB J 2012; 26: 3222-3229

24 Long SB, Hancock PJ, Kral AM, Hellinga HW, Beese LS. The crystal structure of human protein farnesyltransferase reveals the basis for inhibi- tion by CaaX tetrapeptides and their mimetics. Proc Natl Acad Sci U S A 2001; 98: 12948-12953

25 Spartan'14 for Windows, v 1.1.8. Irvine, California: Wavefunction, Inc.; 2014

26 Halgren TA. Merck molecular force field. I. Basis, form, scope, parameterization, and performance of MMFF 94. J Comput Chem 1996; 17: 490-519

received Sept. 25, 2015

revised Sept. 25, 2015

accepted October 21, 2015

Bibliography

DOI http://dx.doi.org/10.1055/s-0035-1558261

Planta Med Lett 2015; 2: e73-e77

(c) Georg Thieme Verlag KG Stuttgart · New York . ISSN 2199-157X

Correspondence

Prof. William N. Setzer

Department of Chemistry

University of Alabama in Huntsville

Huntsville, AL 35899

USA

Phone: +12568246519

Fax: +12568246349

wsetzer@chemistry.uah.edu

\section{Intentram

